\title{
Fabrication of a Lift-Out Grid with Electrical Contacts for Focused Ion Beam Preparation of Lamella for In Situ Transmission Electron Microscopy
}

\author{
Matthew Mecklenburg ${ }^{1}$, Miles Brodie ${ }^{1}$, William Hubbard ${ }^{2,3}$, E. R. White ${ }^{2,3}$, Adam Bushmaker ${ }^{1}$, \\ Erica Deionno ${ }^{1}$, Brendan Foran ${ }^{1}$, and B. C. Regan ${ }^{2,3}$ \\ 1. Microelectronics Technology Department, The Aerospace Corporation, Los Angeles, California \\ 90009, USA \\ 2. Department of Physics and Astronomy, University of California, Los Angeles, California 90095, USA \\ 3. California NanoSystems Institute, University of California, Los Angeles, California 90095, USA
}

The tools used in focused ion beam (FIB) sample preparation of lamella for study via transmission electron microscopy (TEM) are not simply translated to experiments that also require in situ electrical biasing [1]. FIB sample preparation is not well suited for electrically isolated samples because charging of the insulating material isolating the electrical connections makes precision placement of the FIB difficult. In this work we show progress towards making a new type of lift-out grid well suited for in situ electrical biasing experiments, unlike traditional metal grids. Our goal is to design and fabricate a type of electrical biasing grid that will be made commercially available and that will be suitable for in situ electrical testing of a wide range of electronic devices and materials in the TEM.

Lift-out grids for lamella sample preparation are usually 25 to $40 \mu \mathrm{m}$ thick and made from metals such as copper, molybdenum, or beryllium. Half-moon shaped lift-out grids are chosen for two reasons: to facilitate a short FIB working distance (which allows for a more focused beam) and for easier placement of the lamella on the lift-out grid. Our first design of a repeatable process for applying electrical contacts to a lamella is shown in Figure $1 \mathrm{a}-\mathrm{b}$. We initially hoped to coat a metal lift-out grid with $200 \mathrm{~nm}$ of sputtered silicon dioxide, with $30 \mathrm{~nm}$ of Chromium and $100 \mathrm{~nm}$ of Gold placed as contact electrodes via evaporation through a shadow mask. Although a few grids were made this way, mask alignment and evaporating contacts became too time consuming and grids were easily bent while processed making most unusable. Instead of continuing along this direction we decided to switch to a more common substrate for making electrical devices, silicon, and we partnered with an experienced manufacturer of silicon based grids, TEMwindows.com.

Silicon is a better substrate than metal for lift-out grids for electrical biasing because it can be processed on a wafer-sized scale while having the resulting lift-out grids thin enough for placement inside the TEM [2]. A partially suspended $400 \mathrm{~nm}$ thick non-stoichiometric silicon nitride (SiN) membrane was made using a combination of chemical and reactive ion etching and the result is shown in Figure 1c-d. The half-moon shape of the silicon was formed using a $\mathrm{KOH}$ etch. The partially suspended SiN membrane was defined using photolithographic patterning and a reactive ion etch. These grids are as easily placed as their metal counterparts in mounts designed for FIB preparation of lamella, such as the FEI Flip Stage. Figure 2a shows a FIB lift-out transferred lamella mounted near the free edge of the SiN half-window. The lift-out grid's half-moon shape minimized charging effects near the lamella because of its placement near the FIB; the beam hits the sample before interacting with any unneutralized charge on the membrane. Figure $2 \mathrm{~b}$ shows the membrane tilted and the hole cut under the sample (an irregular shape due to charging) to allow imaging with a TEM. The next step is placement of contact electrodes and bond pads on the SiN. The layout of the proposed contacts is shown in Figure $2 \mathrm{c}-\mathrm{d}$. The contacts will allow a single lamella to be connected with up to four terminal connections for electrical biasing of 
FIB transferred materials or devices. Bond pad contacts sit on regions of the grid that still have thick silicon for support and thus will be compatible with wire bonding.

We have developed and tested a new type of grid for preparation of lamella on an insulating material compatible with developed FIB lift-out preparation techniques. This work is the first step to a robust and repeatable way of preparing in situ electrical basing samples for TEM work. The work at The Aerospace Corporation was supported under its Independent Research and Development Program. TEMWindows.com was helpful in developing these silicon based lift-out grids.

References:

[1] J Mayer et al, TEM Sample Preparation and FIB-Induced Damage, MRS Bulletin, 32 (2007), p. 400. [2] B Westenfelder et al, Graphene-based sample supports for in situ high-resolution TEM electrical investigations, Journal of Physics D: Applied Physics, 44 (2011), p. 055502.
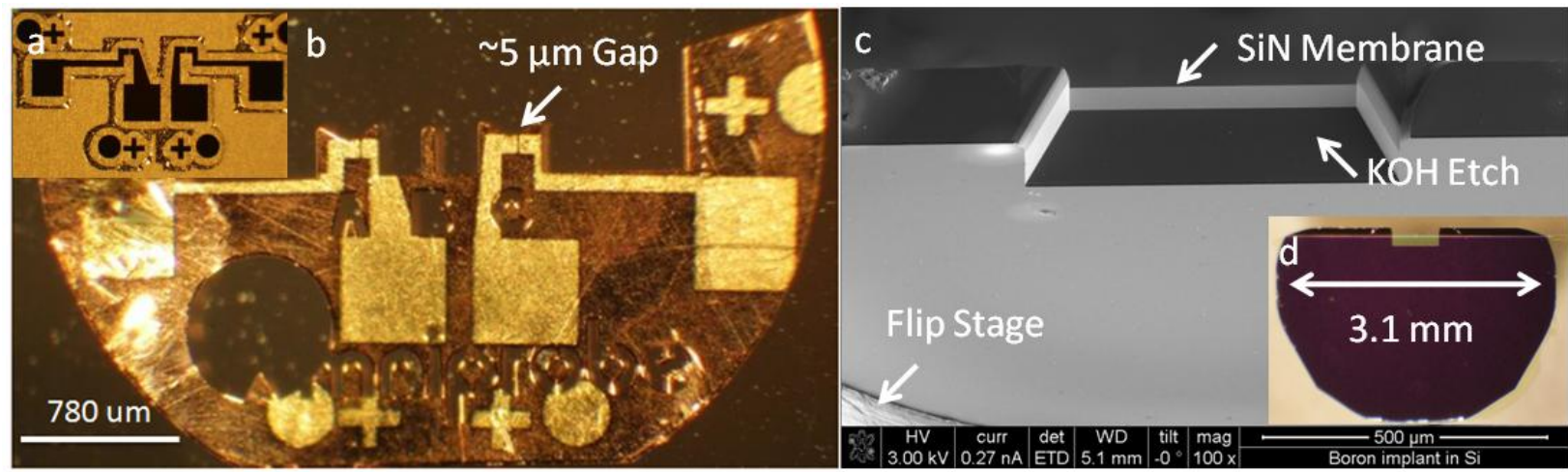

Figure 1. (a) Shadow mask for applying contacts to lift-out grids. (b) Evaporated contacts on a copper Omniprobe ${ }^{\circledR}$ lift-out grid. There is a small gap between the contacts to allow for a sample to be placed between them. (c) The bottom side of the silicon lift-out grid. The $400 \mathrm{~nm}$ thick SIN membrane is clearly shown. The grids are flip stage compatible to facilitate milling with the FIB. (d) An optical image of the grid is shown. The SiN membrane is visible at the top edge of the lift-out grid.
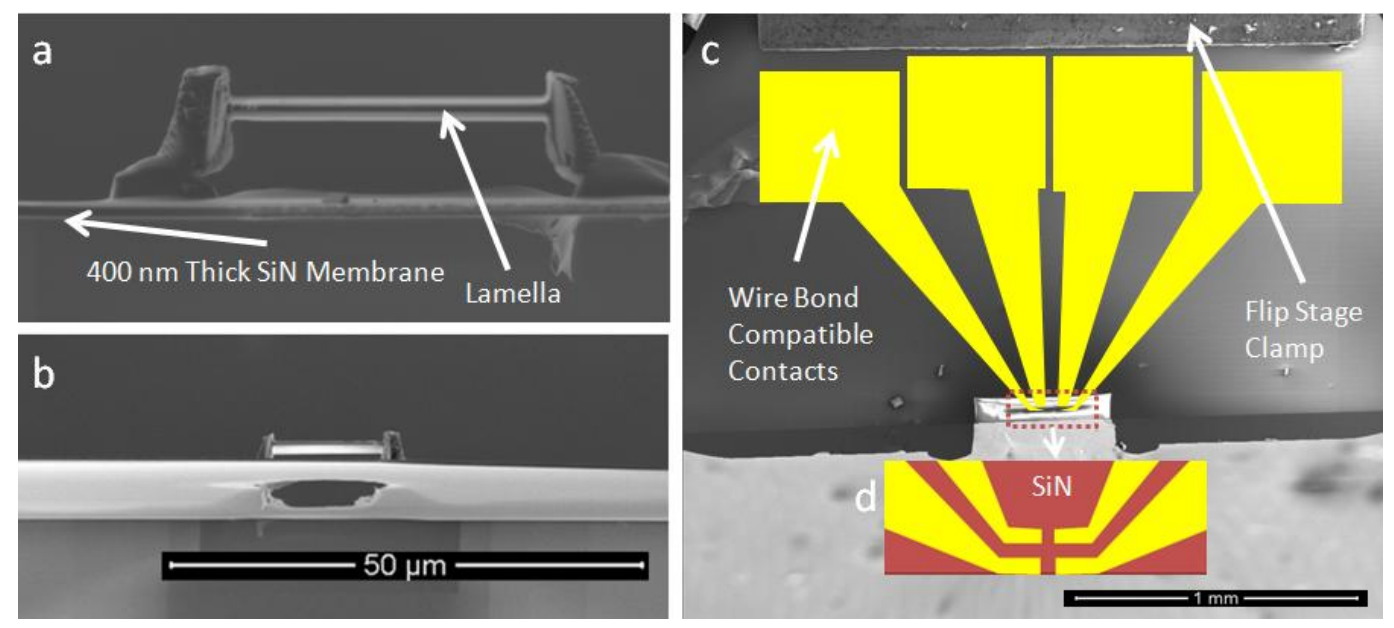

Figure 2. (a) An edge-on view of a FIB milled lamella placed between posts on the SiN membrane. (b) A tilted view of the lamella. The region under the sample was cut to allow for better TEM imaging. (c) Shown is a top down view of the silicon lift-out grid with a schematic of the proposed contacts placed on the sample. (d) An enlarged view of the SiN membrane and the gap between the contacts. 